\title{
Study on College Students' Mental Sub-Health and its Intervention System
}

\author{
Tan Yin \\ Hospital of \\ Qilu University of Technology \\ Jinan, P. R. China \\ tan-su@163.com
}

\begin{abstract}
With the aim to solve college students' mental subhealth problems, this paper discriminated the related concepts such as health, mental health, mental sub-health, analyzed the causes of college students' mental sub-health from themselves, their families, the community, schools, I also constructed an intervention system of college students' mental sub-health, including mental intervention, education intervention and entertainments \& sports intervention in this paper. The results indicate that college students' mental sub-health problems should be taken seriously and the scientific intervention system can help to guide to foster college students' mental health. The main innovation of this paper is to build intervention system for college students' mental sub-health.
\end{abstract}

Keywords-college students; mental sub-health; intervention system

\section{INTRODUCTION}

The relevant statistics of the World Health Organization (WHO) show that more than $80 \%$ of the global population in the state of mental sub-health, the intervention of sub-health research is attracted more and more attention by the theoretical circle and the social public. As Chinese university enrollment expansion, increasing the number of this group of college students at the same time, with the intensification of learning and employment pressure, colleges and universities every year drop out from school, out of school, even the phenomenon of suicide are com-mon. A specific group of college students mental sub-health problem more concern and attention, it is very important to build effective and comprehensive system of college students' mental sub-health intervention.

\section{RELATED CONCEPTS}

\section{A. Health}

The WHO constitution states: "Health is a state of complete physical, mental and social well-being and not merely the absence of disease or infirmity."[1] This definition are widely cited, but there are other definition of health, for example, Bircher (2005) defined it as: "a dynamic state of wellbeing characterized by physical, mental and potential, which satisfies the demands of a life commensurate with age, culture, and personal responsibility."[2] Saracchi (1997) proposed the following definition: "health is referred to under such happiness, no disease, and no weakness, and have basic, universal human rights"[3].

The WHO definition of health is a state of perfect, it is difficult to achieve, and the definition of Bircher is considered the commensurate with age, culture, individual responsibility of health demand, Saracchi incorporated the modern concept of human rights, fairness and justice, etc. into the WHO definition. In short, people's health should include at least three aspects of physical health, mental health and social health.

\section{B. Mental Health}

WHO also give definitions of mental health: "mental health is a state of well-being in which an individual realizes his or her own abilities, can cope with the normal stresses of life, can work productively and is able to make a contribution to his or her community". An important implication of this definition is that mental health is more than just the absence of mental disorders or disabilities.

Health and mental health are all relative concepts, absolute health is non-existent, an individual's health are at a point that in the middle of relatively healthy and unhealthy, and an individual's mental health status is dynamic change, rather than a static motionless. This will naturally have the concepts of "sub-health" and "mental sub-health".

\section{Sub-health and Mental Sub-Health}

Sub-health was first proposed by the former Soviet Union scholar Professor Buchman in the mid 1980's, but no research or explore the connotation and extension of the concept of mental sub-health outside of China, "subliminal obsessivecompulsive disorder" and "subthreshold depression" concept connotation and is similar to it[4].

The concept of mental sub-health in China have discussed more fully, which is representatively defined as mental characteristics in the environmental impact of genetic and congenital conditions of the decision (such as personality, love, emotion, intelligence, endurance, etc.) cause health problems. It is an intermediate state between mental health and mental illness. This is mainly unexplained mental fatigue, emotional disorders, thought disorders, panic, anxiety, low self-esteem as well as nervous, cold, lonely, reckless, even suicidal thoughts and so on. "[5] 
III. The Characterization of College Students' Mental SuB-HEALTH

\section{A. Main Physical Characterization: Neurasthenia}

The main external magnification of mental sub-health is neurasthenia, which is in the clinical characterization of body, this mainly appears as follows: mental fatigue, sleepiness, body weakness; lassitude and low efficiency in the day of the study and memory difficulties; failure to sleep, excessive dreaming and undound slumber at night.

The author has found that the high-risk nervous breakdown period of college students is second semester of sophomore and first semester of junior, during this period, feeling of freshness into the university for students has being in the past, and they are not prepared for the transition from high school to college life, learning, emotion, social activities may encounter all sorts of setbacks, which are easy to cause neurasthenia. Along with neurasthenia, many patients will appear nervous headache.

\section{B. Main Mental Characterization: Depression}

The mental characterization of mental sub-health is depression, which is often accompanied with neurasthenia. Quite a number of college students has overestimated their ability, they have too beautiful vision for a lot of things, Setbacks will be difficult to avoid, once they can't correctly deal with the setbacks, they may go to the other extreme that they are too pessimistic for everything, which shows no confidence for study, no due interest and enthusiasm for extracurricular activities, no hope for personal future, especially with suicidal thoughts.

The main external magnifications of depression includes that volitional activities drop, initiative activities reduced significantly, and they often refuse to participate in the activities of interest to the outside and are often alone; meanwhile, they think slowly and laboriously, speak slowly and incoherently, act slowly and soullessly; their bodies appear gastrointestinal function disorders, accompanied by dizziness, headache, limbs weakness, going limp, and the serious ones may appear cardiac function disorder and feel discomfort from system and organ with varying degrees.

\section{Main Social Characterization: Interpersonal Barriers}

Interpersonal relationship plays a vital role in the mental health, and good interpersonal relationship can relieve mental pressure and promote mental health. A mental sub-healthy person often causes social communication barriers.

The frustration of social interaction is one of the main reasons of mental sub-health for college students, interpersonal barriers has become the main characterization of the patients with mental sub-health. Entering university campus, each university freshmen have strong need extensive exchanges with others, but their communication mode may be improper and their communication ability is limited so that setbacks in interpersonal communication cannot avoid, which will make some students look communication as a burden, they may close themselves gradually and look for comfort on the internet, therefore they won't communicate with classmates, friends and teachers. In addition, a substantial part of college students are introverted, especially the ones from poor families have a strong inferiority mentality, they are afraid of being looked down on in social activities, and they are unwilling to participate in the competitive society. Over time, they are more likely to meet social problems.

\section{The Causes of College Students' Mental Sub-Health}

\section{A. Their Own Reason: The Disorders of Role Conversion and Adjustment}

During a special period of life, college students have the special physiological and mental characteristics. In physiology, contemporary college students generally appear obvious precocity because of the material living condition improvement, while their mental maturity becomes backward, and the conflicts between physiological maturity and the mental immature will induce mental problems.

The freshman must adapt to college life and complete the conversion of role. They are no longer like in high school when they had too many constraints and pressure, their social intercourse become much more, they need to handle the relationships among the inside and outside class; their study should transform from the teachers' instruction to instill independence. Facing the new environment, new relationships and new teaching mode, quite a few students adapt slowly, and thus produce mental confusion, a huge sense of loss, psychological disorders, and low self-esteem or self-enclosed, the text will appear severe mental sub-health.

\section{B. Family Reasons: Excessive Doting and High Expectations}

The family is the first "window" for college students to enter the society and to understand society, it plays vital role in psychology education. But most of the families have tried to meet the children's material needs and ignore their mental health, especially nowadays, most children are the only child in the family and spoiled by the parents, which induces them to develop the habit of willfulness, vanity, selfishness, when they enter the university, it is difficult for them to adapt to the collective life and interpersonal relationships; on the other hand, parents want their children to succeed of course, high expectations for children are mistaken as sided pursuit of test scores, and children don't pay attention to social intercourse and setback education so that they are autistic easily .

Two groups of students should be attached importance: the first ones are from rich families, whose study and living are wishful and can't bear aspects of setbacks, therefore they appear psychological distortion easily once some setbacks happen; the other ones are from poor families, they have great pressure on the economy, with strong psychological selfesteem or low self-esteem, interpersonal relationship is more easy to negative "closed", if the guidance to them is not correct, they are prone to psychological problems.

\section{Social Rreasons:Money Worship and Lack of Faith}

The current college students were born in the acute period of economic transformation in China, which constantly enrich people's material and cultural life and some high-grade products are being geared, the entertainments into the life of 
people are studs or duds, some of them justly meet college students' psychology for novelty and difference, these activities need money, it is easy to produce psychology of money worship. Meanwhile, with the development of market economy and deepening of the reform and opening to the outside world, western ideas, culture and thoughts are spread into the domestic, and there is a lot of dross, social atmosphere is getting worse, filled with money and material desires. College students cannot distinguish and resist the dross, and it is easy for them to occur the distort of world outlook, life outlook and values outlook, inner conflict and confusion will lead them to lack of ideal and faith, lack of positive spirit, lack of social responsibility and social morality so that their study and life have no goal and objective, and tend to self-enclosed, quite a few college students are addicted to the internet and mobile phone, which lead to moral confusion, cognitive dissonance, psychological unbalance and develop mental sub-health.

\section{School Reasons: Improper Education and Lack of Care}

Chinese "exam-oriented education" has repeatedly come under strong criticism, but it is hard to be changed, and entering good junior school has become the goal of children's study and life from the beginning of elementary school, scores and graduation are almost the only guide line for teachers and students, while sound character and good psychological quality have been seldom paid attention to before university. After entering university, the major setting and training mode are helpful to cultivate the students' professional knowledge, professional skills, but college students' mental health is still not under the attention they deserve.

Currently, some unhealthy tendencies have been spread into campus, which lead to competition consumption phenomenon, bureaucratic phenomenon, exam cheating phenomenon, utilitarian phenomenon, violence and underworld phenomenon in campus, which will adversely affect students' mental. It is worth paying attention to that the staffs of student affairs administration have not increase with college students after sharp expansion of university enrollment scale, and there is widespread shortage of manpower, the vast majority of students have not gained enough attention and care, therefore, their psychological fluctuations can't be found in time, although many schools also offer mental health education curriculum, and build mental counseling class institutions, but most of them can't play substantial role, college students' mental sub-health cannot be avoided.

\section{THE CONSTRUCTION OF INTERVENTION SYSTEM FOR College Students' Mental Sub-Health}

College students have been in the face of increasingly common mental sub-health phenomenon, the measures for prevention and intervention should be taken, and it is a longterm, complex systems engineering and need to take a variety of measure because of complexity and unpredictable nature of mental problems, therefore, I construct intervention system, including mental intervention, education intervention and entertainments \& sports intervention.
A. The Mental Intervention for College Students' Mental Subhealth

- Establish and improve the mental health mechanism of college students. Mental health governing bodies and mental health consultation center should be established at school level, mental health consultation workstation should be established in various departments, mental health consultation member should be designated in each class, and mental health consultation liaison should be designated in each dormitory, so that each student's psychological abnormalities can be found in time and it can be reported and dealt with in time. Meanwhile, school hospital or other health care institution should introduce or train professional mental health care staffs and set up mental health department to provide professional guidance and targeted treatment.

- Establish college students' mental health files. In order to improve the mental health of college students, the school should carry out a mental health survey in college students, and set up the mental health record based on the census results, so that the mental characteristics and the mental problems of students can be mastered comprehensively, which can provide the basis for timely detection of mental sub-health phenomenon, and timely mental counseling and counseling or other treatment plans.

- Develop mental counseling in college students. Mental consultation is a very direct and effective way to solve college students' mental sub-health, schools should take it as a routine work. Firstly, the publicity of mental counseling should be strengthened to let all students know it, understand it and then close it; secondly, the skilled excellent mental counseling teachers' team should be established to provide the consolation what students want. Finally, schools should try to facilitate communication and problem consulting for students, especially carry out a variety of mental consulting activities, students can communicate and consult through network, telephone, interviews, and other ways.

\section{B. The Education Intervention for College Students' Mental Sub-health}

- Strengthen the propaganda of the knowledge of mental health so that students are able to understand the mental health of basic knowledge in a variety of ways, grasp their mental state, and master the basic method of selfmental adjustment. Schools can not only start to open the curriculum of mental health education, but also transfer mental health knowledges through pamphlets, campus network, campus radio, seminars.

- Guide college students for correct understanding of self. Students should be guided to understand self from physiology, psychology and society through a variety of ways. Firstly, students should be educated and guided to broaden their knowledge, improve their cultural training and cultural level to enhance all aspects of their quality, which is the premise of cognitive self; Secondly, students should be encouraged to communicate with 
friends, classmates and teachers to know the others' opinion about themselves to avoid excessive selfperception; Finally, students should be guided for understanding of self from their own condition, capacity, status, role and responsibility both in social reality, historical conditions and in a small environment, which is helpful to the appropriate self-positioning and the overall correct understanding of self.

- Educate students to establish a correct outlook on life. Schools should strengthen ideological and political education of students, educate and guide students to establish a correct outlook on life, establish a positive attitude and good moral character, so as to correctly face with unhealthy trends in society, even in this small community on campus, educate and guide students away from and against money worship, hedonism, individualism and other wrong outlook on life, which can provide accurate navigation for the students on the road of life and provide them with psychological activity "positioning system", to lay the solid foundation for them to own good psychological quality.

\section{The entertainments \& sports intervention for College Students' Mental Sub-health}

- Give full play to the role of recreational activities in college students' mental health. Schools should advocate and encourage to hold a variety of recreational activities such as Weekend Theater, concerts, dance, singing contest, calligraphy competition, literature lecture, fiction contest, which can enrich the life of students, and prevent unhealthy things from invading students' amateur life. Especially, it is worth noting that music nursing intervention of patients with mental subhealth is a kind of effective, cheap, and no traumatic easy relaxation method [6]. Therefore, medical workers and student affairs staffs should seriously study and master the music intervention methods and procedures to be able to direct students by music and character cultivation, regulating emotions, and the related medical staffs can take advantage of music on psychological sub-health nursing intervention for students.

- Give full play to the role of sports in college students' mental health. Sports participants are generally required to have physical strength, endurance, and many sports are quite tough, confrontational, and intense. Regular physical activities can exercise willpower, enhance selfconfidence, ease the psychological pressure, and relieve mental anxiety and negative emotions; many sports activities are an important way of social interaction, can increase contacts between people and meet a certain sense of belonging and social needs. Some studies also proved that sport is an effective channel of mental subhealth intervention [7]. In order to promote the mental health of college students, schools must start from the following aspects: (i) to guide students to develop morally, intellectually and physically. Physical education should be given equal importance with moral education, intellectual education, and students should be instructed to participate in all kinds of physical training and sports competition actively by supervision and encouragement. (ii) Schools should actively create the environment and atmosphere of sports for students. Schools must increase investment to build the necessary stadiums, repair and improve all kinds of sports facilities in time, vigorously promote the development of various sports community organizations, through their unity, a group of students are lead to take the initiative to go into sports. Schools and colleges should actively organize various sports activities and sports competitions; organize all kinds of track and field games, and ball games regularly or irregularly. (iii) Sports mental intervention should master several principles: sports should be designed and selected carefully, exercise scientifically; the choice of sports should vary from person to person; collective projects should be emphasized and many people are encouraged to participate in them.

\section{CONCLUSION}

College students are the main force of future construction, their physical and mental health are vital to the future of the motherland and the nation, college students' mental health problems cannot be neglected, the study of college students' mental sub-health is more and more important, more and more urgent, this paper analyzed the reasons including their own reasons, family reasons, social reasons, and school reasons, I also put forwards that mental sub-health intervention is a systematic project in this paper, which requires the integrated use of various means, including at least mental, educational and entertainments \& sports areas measures, of course, these measures intervention system is far from all the mental intervention, this paper might need to be further expanded and refined.

\section{REFERENCES}

[1] http://www.who.int/mediacentre/factsheets/fs220/en/

[2] J. Bircher, "Towards a dynamic definition of health and disease", Med. Health Care Philos,vol. 8, pp. 335-341, 2005

[3] R. Saracci, "The World Health Organization needs to reconsider its definition of Health",British Clinical Research vol.314, pp.1409$1410,1997$.

[4] Xie Dongbei, Lin Ling, "Mental sub-health: the concept of clearance and selection criteria", Modern Preventive Medicine, vol. 35(1), pp.8-9, 2008(In Chinese)

[5] http://baike.baidu.com/view/4960172.htm?fr=aladdin(In Chinese)

[6] Huang Suying, Yangxia, "Music nursing in mental sub-health patients", Modern Nursing, vol. 10(12), pp.1150-1151,2004(In Chinese)

[7] Xie Dongbei, "College Students' Mental Sub-health and its Physical Intervention", Journal of Community Medicine, vol.6(17), pp.52-53, 2008(In Chinese) 